Trivent Publishing

(C) The Authors, 2016

Available online at http://trivent-publishing.eu/

Engineering and Industry Series

Volume Power Systems, Energy Markets and Renewable Energy Sources in

South-Eastern Europe

\title{
Inclusion of Phasor Measurements in the State Estimator of the Serbian TSMO SCADA/EMS System
}

\author{
Miloš Stojić, ${ }^{1}$ Jelena Veselinović, ${ }^{2}$ \\ Ninel Čukalevski, ${ }^{3}$ Goran Jakupović ${ }^{4}$ \\ 1,3,4 "Mihajlo Pupin" Institute, Belgrade, Serbia, estojicm@ rcub.bg.ac.rs \\ 2 "Elektromreža Srbije", Belgrade, Serbia
}

\begin{abstract}
Although nowadays, within the transmission system smart grid efforts, new DSP (Digital Signal Processing) based ultra-fast remote terminal units (known as $P M U)$ are increasingly installed in the power systems, the use of their data is mostly limited to phasor visualization at the system control centre. Within the steady state domain, their first practical use might be within the state estimation (SE) application of the classic SCADA/EMS system. This can be justified due to the importance of SE for all of the real time/study network applications.

Due to their high accuracy and existence of phase angle measurements, PMU measurements offer a potential benefit to the quality of state estimation solution. In Serbian TSMO, a new WAMS system has been implemented with PMU devices installed during the Project phase in several substations. Two classical SCADA/EMS systems are in operation in the Serbian TSMO NCC, where one serves as a primary, while the second one is a backup for SCADA/EMS, but at the same time it serves as a primary one for SMM Block load frequency regulator. The backup system is open for upgrade, so the State Estimator has

This is an Open Access article distributed in accordance with the Creative Commons Attribution Non Commercial (CC-BY-NC-ND 4.0) license, which permits others to copy or share the article, provided original work is properly cited and that this is not done for commercial purposes. Users may not remix, transform, or build upon the material and may not distribute the modified material (http://creativecommons.org/licenses/by-nc/4.0/)
\end{abstract}


been modified to include voltage phase angle measurements, using approach with no reference bus specified. The influence of phase angle measurements on state estimation accuracy has been investigated, using system snapshots taken from the real-time system.

The results of this study and practical consequences for the Serbian TSMO $N C C$ will be presented in this paper, as well as problems identified.

\section{Keywords}

State Estimation; PMU; phasor measurements EMS; Network Topology

\section{Introduction}

In 2015/2016, the Serbian TSMO installed newly-purchased PMU devices, whose locations were strategically chosen as the most important energy points of the Serbian power system. After having all the implementation work and tests done, a new WAMS system emerged on the European power systems chart and new possibilities and challenges imposed for the engineering staff and that is how to make the best use of the information, provided by this system. As already known, measurements from these devices are primarily used for the purpose of dynamic assessments and early warning alerts, but secondly (here, we will work on another aspect of its use), is their use in static power system assessment, which is related to SCADA/EMS systems, which in Serbian TSMO is a double task, knowing the fact that there are two such systems, running in parallel, and also that they are both manufactured by different producers.

Measurements from these PMUs, previously adapted to SCADA/EMS sampling frequencies, were also introduced into both of these SCADA/EMS systems/databases.

As for state estimator (SE), currently, these measurements are used as the redundant measurements for already traditionally used SE measurements and they will be used as the most reliable for the SE on both systems in real time as well, in a while, after the completion of all reliability tests.

However, PMUs also provide some "new" kind of very precise measurements, which are at our disposal, for their best use for even more accurate and reliable state estimation. The most interesting additional information are voltage angles obtained by PMUs and it is exactly what will we try to make a benefit out of, for SE.

Both SCADA/EMS systems in Serbian TSMO are in the process of constant improvement. The leading one is planned for upgrade and its current version of $\mathrm{SE}$ application is not primarily aimed for the use of angle voltages (beside voltage magnitudes), collected off from Serbian "PMU grid." The other one - a backup SCADA/EMS system has been recently upgraded, is in production as well and is currently about to enter the second phase of the upgrade process, so the analysis in this paper relates to tests performed on its software, especially 
since it is more open and flexible for improvements. So far, the newest SE application of this backup system has shown a great robustness to topology errors and by adding many new features has been grossly improved in comparison to its previous versions. These latter improvements, introducing PMU measurements into SE are the main subject of this paper.

As for SE application and SCADA/EMS in general, the goal is to be in the line with modern power grids, which are also in constant upgrade and reconstruction, so developing new ideas and upgrading software and hardware, based on experience and new needs is a must.

\section{Traditional state estimation - theoretical background}

The theory of Weighted Least Squares (WLS) State Estimation (SE) is very well known, as this basic theory for Static Power System SE was developed in the 1970 's and it has been used in Control Centers for decades. The purpose of the $\mathrm{SE}$ is to determine the state vector (vector of complex voltages), which is the best fit to the set of redundant SCADA measurements (over determined problem). Once the state vector is determined, all other physical values in the system are easily calculated. State estimator also serves as filter for bad measurements. Through Bad Data (BD) analysis, bad measurements are identified and eliminated.

The best estimate of the state vector $\mathrm{x}$ is obtained by the Weighted Least Squares (WLS) method. The objective is to minimize the sum of the squares of the weighted residuals (deviations of the estimated measurements from the actual measurements). Cost function written in compact, matrix form is:

$$
\begin{aligned}
& \mathrm{J}(\mathrm{x})=[\mathrm{z}-\mathrm{h}(\mathrm{x})]^{\mathrm{T}} \mathrm{W}[\mathrm{z}-\mathrm{h}(\mathrm{x})] \\
& \mathrm{J}(\mathrm{x})-\operatorname{cost} \text { function } \\
& \mathrm{x}-\text { state vector }(\mathrm{V}, \boldsymbol{\theta}) \\
& \mathrm{z}-\text { measurements vector } \\
& \mathrm{z}=\mathrm{h}(\mathrm{x})+\boldsymbol{\varepsilon} \\
& \boldsymbol{\varepsilon}-\text { measurements error vector }
\end{aligned}
$$

$\mathrm{h}(\mathrm{x})$ - the nonlinear function relating the measurements to the system states

$\mathrm{W}^{-1}$ is the covariance matrix of measurement errors, which reflects the measurement confidence. In the case of independent measurement errors, its inverse matrix $\mathrm{W}$ contains measurement weights on main diagonal:

$$
\begin{aligned}
& \mathrm{W}_{\mathrm{jj}}=1 / \sigma_{\mathrm{j}}^{2} \\
& \sigma-\text { standard deviations which reflect the measurement uncertainty } \\
& \text { Minimization of the cost is achieved by solving: } \\
& \partial \mathrm{J}(\mathrm{x}) / \partial \mathrm{x}=0
\end{aligned}
$$


Since $\mathrm{J}(\mathrm{x})$ is non-linear, the solution is obtained iteratively where in each step a system of linear equations is solved:

$\mathrm{H}(\mathrm{x})^{\mathrm{T}} \mathrm{WH}(\mathrm{x}) \Delta \mathrm{x}=\mathrm{H}(\mathrm{x})^{\mathrm{T}} \mathrm{W} \Delta \mathrm{z}$

Where

$\Delta \mathrm{x}=\mathrm{x}_{\mathrm{k}+1}-\mathrm{x}_{\mathrm{k}}$

$\mathrm{H}=\partial \mathrm{h} / \partial \mathrm{x}$ is the Jacobian matrix of partial derivatives evaluated at $\mathrm{x}=\mathrm{x}_{\mathrm{k}}$

$\mathrm{H}(\mathrm{x})^{\mathrm{T}} \mathrm{WH}(\mathrm{x})$ - gain matrix

$\Delta \mathrm{z}=\mathrm{z}-\mathrm{h}(\mathrm{x})-$ residual vector (differences between measured and estimated values)

$\mathrm{H}(\mathrm{x})^{\mathrm{T}} \mathrm{W} \Delta \mathrm{z}-\mathrm{RHS}$ (right hand side) vector

At each iteration step a new state vector $\mathrm{x}_{\mathrm{k}+1}$ is calculated based on $\Delta \mathrm{x}$

$\mathrm{x}_{\mathrm{k}+1}=\mathrm{x}_{\mathrm{k}}+\Delta \mathrm{x}$

Iterations finish when $\Delta \mathrm{x}$ is within an appropriate tolerance.

In traditional state estimator, one bus is declared as a reference with zero voltage angle, so the number of unknowns is $2 \mathrm{~N}-1$ ( $\mathrm{N}$-number of buses), and it is the dimension of state vector $\mathrm{x}$ and number of columns in Jacobian matrix. Number of rows in Jacobian matrix equals number of measurements.

In conventional state estimation, measurement vector includes voltage magnitudes, active and reactive flows and injections.

\section{The Inclusion of PMU voltage angle measurements in state estimation}

Several approaches have been investigated in order to include PMU measurements in existing implementation of the State Estimator.

In traditional state estimation, all bus voltage angles are estimated, respecting the chosen reference bus. On the other hand, PMU devices deliver absolute voltage angles measured at the given time instant. Direct inclusion of such measurements would provide unrealistic results. One solution is to install one PMU unit at the reference bus and to use angle measurements with subtracted measured reference angle. Problem with this approach is its inability to detect error of angle in reference bus. Errors in reference angle measurements must be specially treated and detected, prior to normal bad data analysis [1]. This requires development and inclusion of new special software module.

Another possible approach, is to divide state estimation problem in two steps [2]. In the first step, only non-synchronized SCADA measurements are processed, using traditional SE. Using results from the first step, along with the PMU measurements, the state estimation solution is further improved, by post processing using linear estimation.

As the most appropriate and most elegant solution for inclusion of PMU voltage angle measurements, it is chosen to use an approach with no reference 
bus [3]. All voltage angle measurements are directly used and no special treatment for the reference bus is needed. Using this approach, errors in all voltage angle measurements are detectable using existing bad data analysis.

\section{The implementation of PMU measurements in the Serbian TSMO state estimator}

Although the SCADA/EMS system was open for changes and upgrades, given the system complexity, the goal was to minimize changes in software in order to reduce the risk of compromising existing software stability and accuracy. PMU units offer measurements traditionally used in state estimation with addition of voltage angles and complex currents. Some of these physical values are mutually dependent and calculated internally by PMU units. Using current measurements in state estimation may lead to numerical difficulties and these measurements are not supported by our implementation of state estimator. It is decided to use only voltage magnitudes and angles together with MW and MVAr PMU measurements. Implementation of traditional PMU measurements (kV,MW,MVAr) only requires entering higher weighting factors to reflect higher accuracies of these measurements.

On the other hand, inclusion of voltage angle measurements requires following changes in the code of the state estimator:

1. The measurement Jacobian has to include columns corresponding to all bus voltage magnitudes and phase angles. As a result, the dimension of the system state vector must be expanded to include twice the number of buses $(2 \mathrm{~N}$ instead of 2N-1). This applies to number of columns in Jacobian matrix and many auxiliary matrices, existing in the code. All these dimensions had to be changed. Although trivial, these changes were the most critical and time consuming, since the code was written in $\mathrm{C}++$, i.e. without automatic check for exiting out of arrays' boundaries.

2. Inclusion of voltage angle measurements adds rows into the measurement Jacobian. Jacobian is modified, so that for each angle measurement, a partial derivative of angle measurement with respect to angle variable is added, in the corresponding row as the only nonzero term.

Since the voltage angle is directly measured, this only nonzero term is simply:

$$
\partial \mathrm{h}_{\mathrm{i}} / \partial \theta_{\mathrm{i}}=1
$$

The term is added at i-th coulmn in Jacobian which corresponds to the angle measurement.

3. Corresponding elements to RHS vector have to be added as well, since new elements in residual vector $\Delta \mathrm{z}$ appear. 
4. A new mechanism must be implemented in software, for treatment of islands without voltage angle measurements. If the system is divided in several electrical islands or not fully observable and divided in several observable islands, an additional check must be done for the existence of at least one voltage angle measurement in each electrical/observable island. If no such measurement exists, this island has to be estimated in a traditional way by introducing single arbitrary reference bus.

5. Various other changes are needed, due to introduction of voltage angle measurements, like changes in application database, reports generation, software configuration parameters etc. Several new configuration parameters are introduced. Now, it is possible to configure separately whether traditional PMU measurements are used or not, and whether voltage angle measurements are used.

\section{The evaluation of PMU measurement effects on real-time SE results}

Unfortunately, when these effects were evaluated, existing real-time SE database had been in some transitional stage, so the state estimator was not well tuned. A data sample taken from the real time is used for evaluation of PMU measurements influence. For reliable conclusions, it would be necessary to have a well tuned SE with more PMU measurements and a larger number of real-time data samples, so this evaluation is only preliminary, with a main purpose to confirm proper software implementation and existence of beneficial effects of angle measurements to SE.

Due to convergence problems, caused by large number of measurement errors and most likely large number of topological errors, only healthy part of the network was analyzed. The EMS system features a mechanism for network reduction [4], which was used to reduce the system to the more healthy part and achieve estimation convergence. Enhanced Givens method was used for solving state estimation problem [5]. All non PMU measurements have standard deviations $\sigma=0.02$ p.u. (2MW, $2 \mathrm{MVAr}$ or $0.02 \%$ of nominal voltage).

The analyzed (reduced) system has the following dimension:

Connectivity nodes: 2640

Electrical (topological) nodes: 289

Switching devices: 2398

Lines: 247

Transformers: 81

Analog measurements: 2182

A number of installed PMU devices in the test system is relatively small, voltage angle measurements are available in only four buses, all at $400 \mathrm{kV}$ in the following substations:

- Djerdap1

- Sremska Mitrovica2 
- Subotica3

- Nis2

An important quantitative parameter for performance measurement is a normalized cost function. It is a cost function, divided by the degree of freedom, which is the number of measurements minus number of unknowns $J /(m-2 N)$. Bad data analysis is performed by successive elimination of measurements with the largest normalized residuals. Since bad data analysis may reject bad measurements differently due to PMU measurements introduction, it is important to keep track of the exact list of rejected measurements to be able to compare cost functions with identical final measurement sets or alternately to compare normalized cost functions prior to Bad Data analysis.

Another performance index used, is the average percentual deviation of estimated and measured values (\%errors=abs((meas-est)/est)*100). MW and MVAr measurements smaller than 0.2p.u. are not taken into account. It is calculated for all measurements, for all MW and MVAr flows and only for $400 \mathrm{kV}$ flows (most important).

Table 1. Measured and estimated voltage angles

\begin{tabular}{|l|l|l|}
\hline Bus & $\begin{array}{l}\text { Measured } \\
\text { angle }\left[{ }^{\circ}\right]\end{array}$ & $\begin{array}{l}\text { Estimated } \\
\text { angle }\left[{ }^{\circ}\right]\end{array}$ \\
\hline Djerdap1/400kV & 177.82 & 177.82 \\
\hline Nis2/400kV & 165.03 & 165.75 \\
\hline Sr.Mitr.2/400kV & 162.85 & 163.55 \\
\hline Subotica3/400kV & 159.81 & 159.50 \\
\hline
\end{tabular}

The first logical step in the evaluation of angle measurements effect on SE was the verification that estimated angles match the measured ones. The easiest way was to leave only one arbitrary chosen angle measurement and then to perform state estimation. In that case SE reduces to the traditional one with "reference bus" and no angle measurements. Such estimated voltage angles should match the measured ones. One has also to make sure that all angle measurements are in the same observable island. Arbitrary chosen reference bus was in Djerdap1. The results are shown in table 1. It can be seen that all differences between estimated and measured angles are within one degree.

The effects of PMU voltage angle measurements have been analyzed for different standard deviations used for angle measurements. The results are shown in table 2. The first column is a baseline, containing estimation results without PMU voltage angle measurements. Deviations of estimated and measured values are shown only for estimation after BD analysis.

Only in cases where $\sigma=0.005$ and $\sigma=0.002$ for PMU voltage angles, BD analysis rejects exactly the same measurements as in the case with no PMU measurements. So, only in these cases, it makes sense, to compare cost functions 
after the BD analysis. That is why normalized cost functions prior BD are also shown. It can be seen, that the improvement in normalized cost function, due to introduction of angle measurements is less than $1 \%$ and that it is not influenced much by PMU measurements weights.

Table 2. SE performance indexes with included voltage angle measurements

\begin{tabular}{|c|c|c|c|c|c|c|c|c|}
\hline & $\begin{array}{l}\text { With- } \\
\text { out } \\
\text { PMU }\end{array}$ & $\begin{array}{l}\sigma= \\
0.005 \\
{[\mathrm{rad}]}\end{array}$ & $\begin{array}{l}\sigma= \\
0.002 \\
{[\mathrm{rad}]}\end{array}$ & $\begin{array}{l}\sigma= \\
0.001 \\
{[\mathrm{rad}]}\end{array}$ & $\begin{array}{l}\sigma= \\
0.0005 \\
{[\mathrm{rad}]}\end{array}$ & $\begin{array}{l}\sigma= \\
0.0001 \\
{[\mathrm{rad}]}\end{array}$ & $\begin{array}{l}\sigma= \\
0.0000 \\
5 \\
{[\mathrm{rad}]}\end{array}$ & $\begin{array}{l}\sigma= \\
0.000 \\
01 \\
{[\mathrm{rad}]}\end{array}$ \\
\hline $\begin{array}{l}\text { Norm.Cos } \\
\text { t Prior BD }\end{array}$ & 1051.7 & 1043.4 & 1043.4 & 1043.5 & 1043.7 & 1044.1 & 1044.2 & $\begin{array}{l}1044 . \\
2\end{array}$ \\
\hline $\begin{array}{l}\text { Norm.Cos } \\
\text { t After } \\
\text { BD }\end{array}$ & 0.7523 & 0.7467 & 0.7557 & 0.7074 & 0.7276 & 0.7387 & 0.7204 & $\begin{array}{l}0.720 \\
7\end{array}$ \\
\hline $\begin{array}{l}\text { Rejected } \\
\text { meas. }\end{array}$ & 201 & 201 & 201 & 211 & 210 & 210 & 212 & 212 \\
\hline $\begin{array}{l}\text { Averall } \\
\text { meas./est. } \\
\text { dev.[\%] }\end{array}$ & 1.9548 & 1.9302 & 1.9089 & 1.6081 & 1.5978 & 1.5653 & 1.6346 & $\begin{array}{l}1.635 \\
0\end{array}$ \\
\hline $\begin{array}{l}\text { MW flow } \\
\text { meas./est. } \\
\text { dev. [\%] }\end{array}$ & 1.5558 & 1.5507 & 1.5598 & 1.1582 & 1.1577 & 1.1491 & 1.2079 & $\begin{array}{l}1.208 \\
6\end{array}$ \\
\hline $\begin{array}{l}\text { MVAr } \\
\text { flow } \\
\text { meas/est } \\
\text { dev.[\%] }\end{array}$ & 3.8247 & 3.8068 & 3.6692 & 3.3093 & 3.2613 & 3.1418 & 3.2814 & $\begin{array}{l}3.281 \\
1\end{array}$ \\
\hline $\begin{array}{l}\text { 400kV } \\
\text { MW flow } \\
\text { meas/est. } \\
\text { dev.[\%] }\end{array}$ & 0.8356 & 0.8462 & 0.9111 & 0.8006 & 0.8181 & 0.8219 & 0.7599 & $\begin{array}{l}0.761 \\
4\end{array}$ \\
\hline $\begin{array}{l}\text { 400kV } \\
\text { MVAr } \\
\text { flow } \\
\text { meas/est. } \\
\text { dev.[\%] }\end{array}$ & 3.4229 & 3.4404 & 3.4110 & 2.3333 & 2.2826 & 2.0951 & 2.3391 & $\begin{array}{l}2.339 \\
0\end{array}$ \\
\hline
\end{tabular}

On the other hand, the percentual difference between estimated and measured values varies a little more with voltage angle measurements weights. In cases were exactly the same measurement set is used for final estimation after BD analysis, overall measured/estimated deviation is reduced from $1.9548 \%$ to $1.9089 \%$. Higher PMU angle measurements weights pushes further rejection of bad measurements (from 201 to 212 rejected measurements), resulting in more substantial decrease in deviation of estimated and measured values. The best 
results are obtained for $\sigma=0.0001(\mathrm{rad})$ for voltage angle measurements, overall deviation is reduced from $1.9548 \%$ to $1.5653 \%$.

Largest errors (deviations) in state estimation results are in reactive powers, probably due to errors in transformers regulation data. This is where the introduction of PMU voltage angle measurements has the most beneficial effect. In case where angle measurements have $\sigma=0.0001$, reduction of reactive flow deviations at $400 \mathrm{kV}$ is from $3.4229 \%$ to $2.0951 \%$. Positive effects on active power flow deviations are less pronounced.

\section{Conclusion}

Paper presents steps of implementation of PMU voltage angle measurements into state estimator in Serbian national control centre. It is shown on one sample taken from real-time, that introduction of small number of PMU voltage angle measurements has modest beneficial effects on SE results. Most pronounced effects are on reactive power flows, where otherwise the SE has the largest errors. For definite conclusions about PMU measurements' beneficial effects on SE, it is necessary to conduct such analysis on a well tuned SE, with more PMU measurements and a larger number of real-time data samples

\section{References}

[1] I. Slutsker, S. Mokhtari, L. Jaques, J. Provost, M. Perez, J. Sierra, F. Gonzalez, and J. Figueroa. Implementation of phasor measurements in state estimator at Sevillana de Electricidad, in IEEE Power Industry Computer Application Conference Proceedings, May 1995, pp. 392-398.

[2] M. Zhou, V.A. Centeno, J.S. Thorp, A.G. Phadke. An alternative for including phasor measurements in state estimators, IEEE Trans. Power Syst. 21 (4) (2006) 1930-1937.

[3] Jun Z, Abur A. Effect of phasor measurements on the choice of reference bus for state estimation. In: IEEE Power Eng Soc General Meeting, 2004. p. 1-5.

[4] M. Stojić, G. Jakupović, N. Čukalevski. One Implementation of Network Topology Processor Application for the TSO Back-up Control Center, DEMSEE 2011 6th International Workshop on Deregulated Electricity Market Issues in South-Eastern Europe, 20.-21. September 2011, Bled, Slovenia.

[5] N. Vempati, I. W. Slutsker, W. F. Tinney. Enhancements to Givens Rotations for Power System State Estimation. IEEE Transactions on Power Systems, May, 1991. 